\title{
Erratum: Local Structure of Lead-containing Mixed-ion Perovskite Ferroelectrics Studied Using Neutron Total Scattering Analysis
}

\author{
[J. Korean Phys. Soc. 61, 80 (2012)] \\ I.-K. JEONG* \\ Department of Physics Education \& Research Center for Dielectrics and Advanced Matter Physics, \\ Pusan National University, Busan 609-735, Korea \\ Dhananjai PANDEY \\ School of Materials Science and Technology, Institute of Technology, Banaras Hindu University, Varanasi, India
}

DOI: $10.3938 /$ jkps.61.502

The acknowledgement was submitted incorrectly. It is corrected from 'This work was supported for two years by a Pusan National University Research Grant. Work at Los Alamos was carried out under the auspices of the US DOE/Office of Science. This work has benefited from the use of NPDF at the Lujan Center at Los Alamos Neutron Science Center, funded by the DOE Office of Basic Energy Sciences and the Los Alamos National Laboratory funded by Department of Energy under contract W-7405-ENG-36. The upgrade of the NPDF has been funded by the NSF through grant DMR 00-76488.' to 'This work was supported for two years by a Pusan National University Research Grant. Neutron diffraction measurements have benefitted from the use of NPDF beam-line at the Lujan Center at Los Alamos Neutron Science Center, funded by the DOE Office of Basic Energy Sciences.'. 